\title{
COPD promotes migration of A549 lung cancer cells: the role of chemokine CCL2 I
}

\author{
This article was published in the following Dove Press journal: \\ International Journal of COPD \\ 20 May 2016 \\ Number of times this article has been viewed
}

\author{
Barbara Kuźnar-Kamińska' \\ Justyna Mikuła-Pietrasik ${ }^{2}$ \\ Patrycja Sosińska \\ Krzysztof Książek ${ }^{2}$ \\ Halina Batura-Gabryel' \\ 'Department of Pulmonology, \\ Allergology and Respiratory \\ Oncology, ${ }^{2}$ Department of \\ Pathophysiology, Poznań University \\ of Medical Sciences, Poznań, Poland
}

Correspondence: Barbara Kuźnar-

Kamińska

Department of Pulmonology,

Allergology and Respiratory Oncology,

Poznań University of Medical

Sciences, Szamarzewskiego 84 Street,

Poznań 60-569, Poland

$\mathrm{Tel} / \mathrm{fax}+486$ I84I 706।

Email kaminska@ump.edu.pl

\begin{abstract}
Patients with COPD develop lung cancer more frequently than healthy smokers. At the same time, molecular mediators promoting various aspects of cancer cell progression are still elusive. In this report, we examined whether COPD can be coupled with increased migration of non-small-cell lung cancer cells A549 and, if so, whether this effect may be related to altered production and activity of chemokines CCL21, CXCL5, and CXCL12. The study showed that the migration of A549 cells through the polycarbonate membrane and basement membrane extract toward a chemotactic gradient elicited by serum from patients with COPD was markedly higher as compared with serum from healthy donors. The concentration of CCL21 and CXCL12, but not CXCL5, in serum from patients with COPD was also increased. Experiments in which CCL21- and CXCL12-dependent signaling was blocked revealed that increased migration of the cancer cells upon treatment with serum from patients with COPD was mediated exclusively by CCL21. Collectively, our results indicate that COPD may contribute to the progression of lung cancer via CCL21-dependent intensification of cancer cell migration.
\end{abstract}

Keywords: chemokines, COPD, lung cancer, migration

\section{Introduction}

A considerable body of evidence has been accumulated, which shows that COPD predisposes to the development of lung cancer. ${ }^{1}$ At the same time, the mechanism underlying increased incidence of cancer in patients with COPD is still not fully explained. It is generally believed that an important role in this field may be played by chronic inflammation, during which numerous cytokines, chemokines, and growth factors exert stimulatory action toward various aspects of cancer cell progression. $^{2-4}$

One of the most fundamental elements of cancer cell invasiveness, especially in metastatic tumors, is their ability to migrate. ${ }^{5,6}$ When it comes to COPD, the course of the pathology includes increased secretion of various agents, the activity of which may, at least theoretically, promote the migration of lung cancer cells. ${ }^{7,8}$ In recent years, much attention has been paid to the role of chemokines as mediators of cancer cell migration, for example, chemokines CCL21, CXCL5, and CXCL12 have been found to contribute to the migratory properties of breast cancer cells. ${ }^{9-11}$ The same chemokines are known to mediate various elements of lung cancer cell progression, including proliferation, ${ }^{12,13}$ adhesion, ${ }^{14}$ and invasion. ${ }^{15}$ At the same time, their role in the migration of lung cancer cells, especially in patients with COPD, remains unknown.

This study was designed to examine whether serum obtained from patients with COPD with various smoking status (smokers, former smokers, nonsmokers) and 
obstruction stage (according to Global Initiative for Chronic Obstructive Lung Disease [GOLD] criteria) may stimulate the migration of non-small-cell lung cancer cells (A549) in vitro and, if so, whether this effect may be mediated by chemokines CCL21, CXCL5, and/or CXCL12.

\section{Materials and methods}

\section{Chemicals}

Unless otherwise stated, all reagents were purchased from Sigma-Aldrich Co. (St Louis, MO, USA). Tissue culture plastics were from Nunc (Roskilde, Denmark).

\section{Blood samples}

The experiments were performed using serum samples obtained from 113 patients with diagnosed COPD. The disease was confirmed according to GOLD criteria, which encompassed a clinical signature and a spirometric confirmation of airway obstruction (post-bronchodilator forced expiratory volume in 1 second $\left(\mathrm{FEV}_{1}\right)$ /forced volume vital capacity $($ FVC) ratio $<0.7)$. Patients were clinically stable (without disease exacerbation treated with antibiotics and systemic glucocorticosteroids for at least 6 weeks prior to the study). Exclusion criteria included inability to perform the spirometric examination, asthma, tuberculosis, lung interstitial diseases, pulmonary thromboembolism, and other chronic pulmonary diseases. In addition, the experiments included 44 healthy age-matched volunteers. The demographic characteristics of the patients and control individuals are presented in Table 1 . The blood samples were collected as follows: $20 \mathrm{~mL}$ of whole blood from patients with COPD and healthy volunteers was collected in the morning (between $6.00 \mathrm{am}$ and $8.00 \mathrm{am}$ ) into EDTA tubes, and then it was centrifuged at $4,000 \mathrm{rpm}$ for 15 minutes at $4^{\circ} \mathrm{C}$. The serum was stored at a temperature of $-80^{\circ} \mathrm{C}$ until used. The study was approved by Poznan University of Medical

Table I Characteristics of COPD patients and healthy volunteers (control group)

\begin{tabular}{lll}
\hline & $\begin{array}{l}\text { Patients with } \\
\text { COPD }(\mathbf{n}=1 \text { I 3) }\end{array}$ & $\begin{array}{l}\text { Control } \\
\text { group }(\mathbf{n}=\mathbf{4 4})\end{array}$ \\
\hline Male/female (n) & $78 / 35$ & $27 / 17$ \\
Age (years; mean \pm SD) & $66 \pm 8$ & $6 \mathrm{I} \pm 9$ \\
Smoking status (n) current/ & $40 / 70 / 3$ & $\mathrm{I3/II/20}$ \\
former/nonsmoker & & \\
GOLD obstruction stage & & \\
$\quad$ Mild (n/\%) & $4 / 3.54$ & $\mathrm{~N} / \mathrm{A}$ \\
Moderate (n/\%) & $22 / 19.47$ & $\mathrm{~N} / \mathrm{A}$ \\
Severe (n/\%) & $43 / 38.06$ & $\mathrm{~N} / \mathrm{A}$ \\
Very severe (n/\%) & $44 / 38.93$ & $\mathrm{~N} / \mathrm{A}$ \\
\hline
\end{tabular}

Abbreviations: SD, standard deviation; GOLD, Global Initiative for Chronic Obstructive Lung Disease; NA, not available.
Sciences Ethics Committee, and all patients included in the study gave their written informed consent.

\section{Cancer cell line}

The lung cancer cell line A549 was purchased from the American Type Culture Collection (Manassas, VA, USA) and propagated in Roswell Park Memorial Institute (RPMI)1640 medium supplemented with $10 \%$ fetal bovine serum and antibiotics.

\section{Measurement of cancer cell migration}

Cancer cell migration through a polycarbonate membrane ( $8 \mu \mathrm{M}$ pores) toward the chemotactic gradient generated by the serum from patients with COPD and from healthy controls was examined using ChemoTx chambers (Neuro Probe, Gaithersburg, MD, USA). In brief, the cancer cells were probed with calcein-AM ( $5 \mu \mathrm{M}, 45$ minutes, $\left.37^{\circ} \mathrm{C}\right)$, and then $4 \times 10^{4}$ cells were suspended in serum-free growth medium and applied onto the top side of the filter to form a hemispherical drop. Afterward, the cells were allowed to migrate toward the sera for 60 minutes at $37^{\circ} \mathrm{C}$. After incubation, the membranes with cells that did not migrate were removed, and the intensity of fluorescence emitted by the cells that did migrate through the membranes was recorded using the Wallac Victor 2 spectrofluorimeter (PerkinElmer Inc., Waltham, MA, USA). The results of migration obtained using ChemoTx chambers were verified using Cultrex BME Cell Invasion Assay (R\&D Systems, Inc., Minneapolis, MN, USA), in which the cancer cells probed with calcein-AM migrated through the basement membrane extract toward the sera in the same conditions as described earlier.

In some experiments, the ChemoTx chambers were used to assess migration toward serum samples preincubated with specific neutralizing antibodies against CCL21 $(2 \mu \mathrm{g} / \mathrm{mL}$; R\&D Systems, Inc.), a specific inhibitor of the receptor for CXCL12 (AMD3100, $1 \mu \mathrm{g} / \mathrm{mL}$; Sigma-Aldrich Co.), or both. The neutralization procedure was performed on a vertical rotator for 1 hour at $37^{\circ} \mathrm{C}$.

\section{Determination of CCL2 I, CXCL5, and CXCLI 2 concentration}

The concentration of CCL21, CXCL5, and CXCL12 in the serum samples was measured using specific DuoSet ${ }^{\circledR}$ Immunoassay Development kits (R\&D Systems, Inc.) according to the manufacturer's instructions.

\section{Statistics}

The results were analyzed using GraphPad Prism v.5.0 software. The groups were compared with the Mann-Whitney 
test or with repeated measures of analysis of variance followed by the Newman-Keuls test as the post hoc. Differences with a $P$-value $<0.05$ were considered significant.

\section{Results and discussion}

Although the pathogenesis of lung cancer is well recognized, ${ }^{16}$ very little is known about the molecular background of increased incidence of pathology in patients with COPD. Interestingly, both pathologies are closely associated with chronic inflammation during which several agents, mostly of a proinflammatory nature, are produced at increased level and contribute to various elements of cancer progression. ${ }^{7}$ One of these elements is the process of cancer cell migration, the role and mediators of which have been poorly explored in the context of the relationship between lung cancer and COPD. ${ }^{17}$ In this report, we addressed this issue by verifying whether COPD may promote the migration of lung cancer cells and, if so, whether this effect may be mediated by altered production and activity of three chemokines: CCL21, CXCL5, and CXCL12.

The experiments were performed using A549 non-smallcell lung cancer cells that were chosen as an experimental model due to their similarity with pulmonary alveolar type II cells and because non-small-cell lung cancer constitutes $\sim 85 \%-90 \%$ of lung neoplasms. ${ }^{18,19}$ The choice of CCL21, CXCL5, and CXCL12 chemokines was, in turn, dictated by recent findings pointing to their critical role in the migration of breast cancer cells ${ }^{9-11}$ and in the progression of lung cancer cells. ${ }^{12-15}$ It is worth noting that the receptors for all these chemokines, that is, CCR7 for CCL21, CXCR2 for CXCL5, and CXCR4 for CXCL12, are known to be constitutively expressed on the surface of A549 cells. ${ }^{20-22}$
The experiments showed that, indeed, A549 cells subjected to serum from patients with COPD migrated more effectively than those exposed to serum from healthy volunteers. This effect was evident when the cells migrated either through the polycarbonate membrane $(P<0.0001)$ or through the basement membrane extract $(P<0.0001)$, thus providing a view on the broader process of cancer cell invasion (Figure 1A). Interestingly, when the results of migration were confronted with the smoking status of both groups of serum donors as well as with the obstruction stage in the patients with COPD, we found that increased migration of the cancer cells was to the largest extent determined by active smoking (Figure 1B) and advancement of the disease (Figure 1C).

Having established these differences, we compared the concentration of CCL21, CXCL5, and CXCL12 in both groups of sera. The results showed that the concentration of CCL21 (Figure 2A) and CXCL12 (Figure 4A), but not CXCL5 (Figure 3A), in the serum from patients with COPD was significantly higher than in the control group $(P=0.0002$, $P=0.0001$, respectively). When these results were re-analyzed with respect to smoking status, the greatest levels of chemokines were recorded for current smokers (CCL21 and CXCL12) and former smokers (CXCL12) (Figures 2B, 3B, and 4B). This finding, in combination with analogical results regarding the migration process, indicates that active smoking is a major determinant of lung cancer cell progression in patients with COPD. Patients who did not smoke, or at least had stopped smoking some time ago, seemed to be relatively safe from the cancer development. This observation is consistent with the findings of other groups who discovered that the risk of lung cancer development and mortality rate from
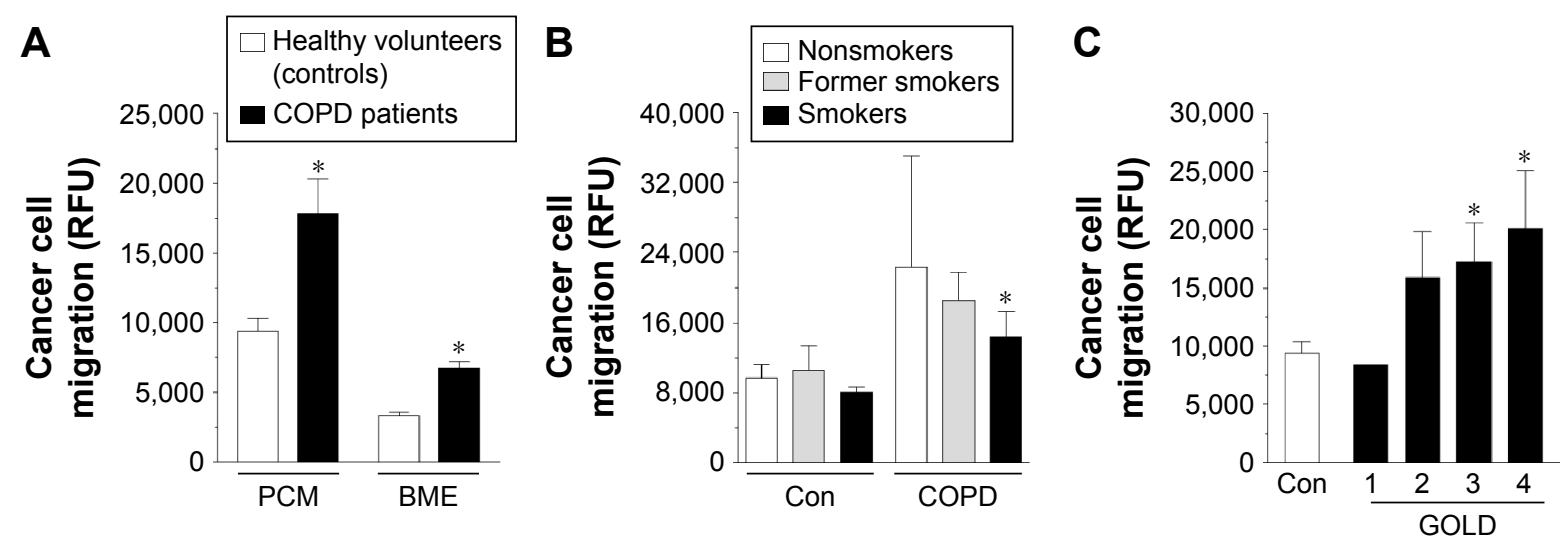

Figure I Efficiency of lung cancer cell migration toward the chemotactic gradient generated by serum from patients with COPD and from healthy volunteers (Con). Notes: Cancer cell motility was evaluated in two experimental systems, namely, through the PCM and through the BME (A). Migration of the cancer cells in reference to smoking status (B) and obstruction stage $(\mathbf{C})$. The asterisks indicate a significant difference as compared with the control group. The experiment was performed with sera from 32 patients with COPD and from 32 healthy donors. The results are expressed as mean \pm SEM.

Abbreviations: BME, basement membrane extract; Con, control; GOLD, Global Initiative for Chronic Obstructive Lung Disease; PCM, polycarbonate membrane; RFU, relative fluorescence units; SEM, standard error of the mean. 
A

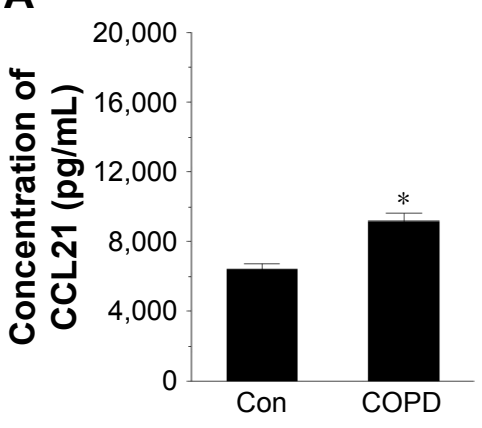

B

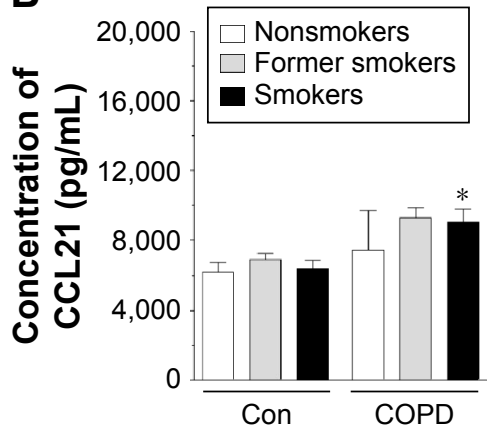

C

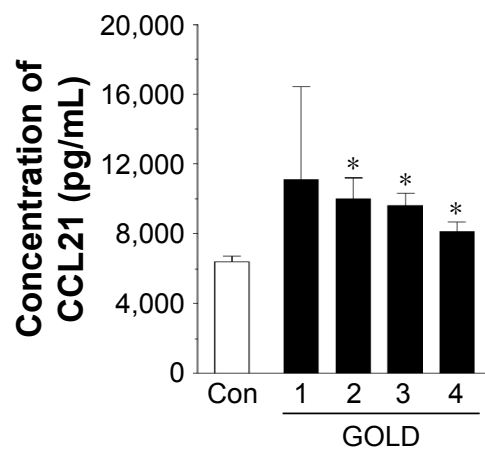

Figure 2 Effect of COPD on the concentration of chemokine CCL2I in blood serum.

Notes: The chemokine level was analyzed in serum samples from all patients with COPD and healthy donors (A) as well as from donors divided according to smoking status (B) and obstruction stage (C). The asterisks indicate a significant difference as compared with the Con group. The results are derived from the chemokine measurements in sera obtained from II 3 patients with COPD and from 44 healthy volunteers. The results are expressed as mean \pm SEM.

Abbreviations: Con, control; GOLD, Global Initiative for Chronic Obstructive Lung Disease; SEM, standard error of the mean.

A

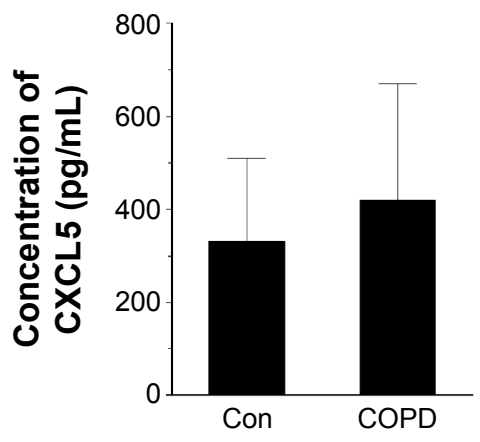

B

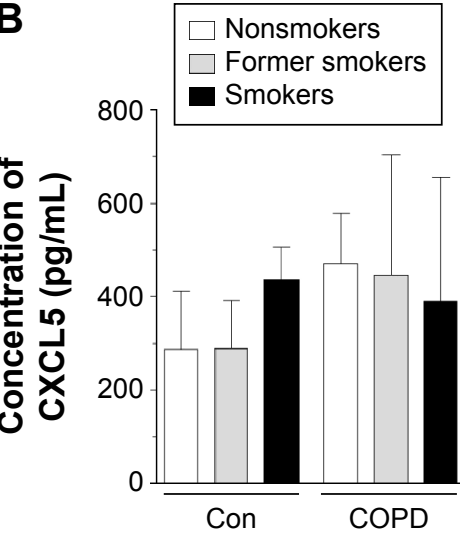

C

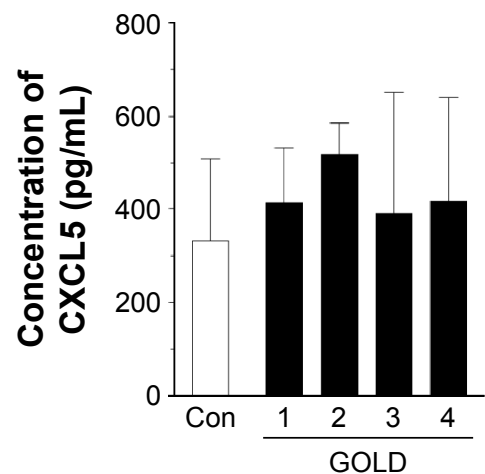

Figure 3 Effect of COPD on the concentration of chemokine CXCL5 in blood serum.

Notes: The chemokine level was analyzed in serum samples from all patients with COPD and healthy donors (A) as well as from donors divided according to smoking status (B) and obstruction stage (C). The results are derived from the chemokine measurements in sera obtained from II 2 patients with COPD and from 44 healthy volunteers. The results are expressed as mean \pm SEM.

Abbreviations: Con, control; GOLD, Global Initiative for Chronic Obstructive Lung Disease; SEM, standard error of the mean.
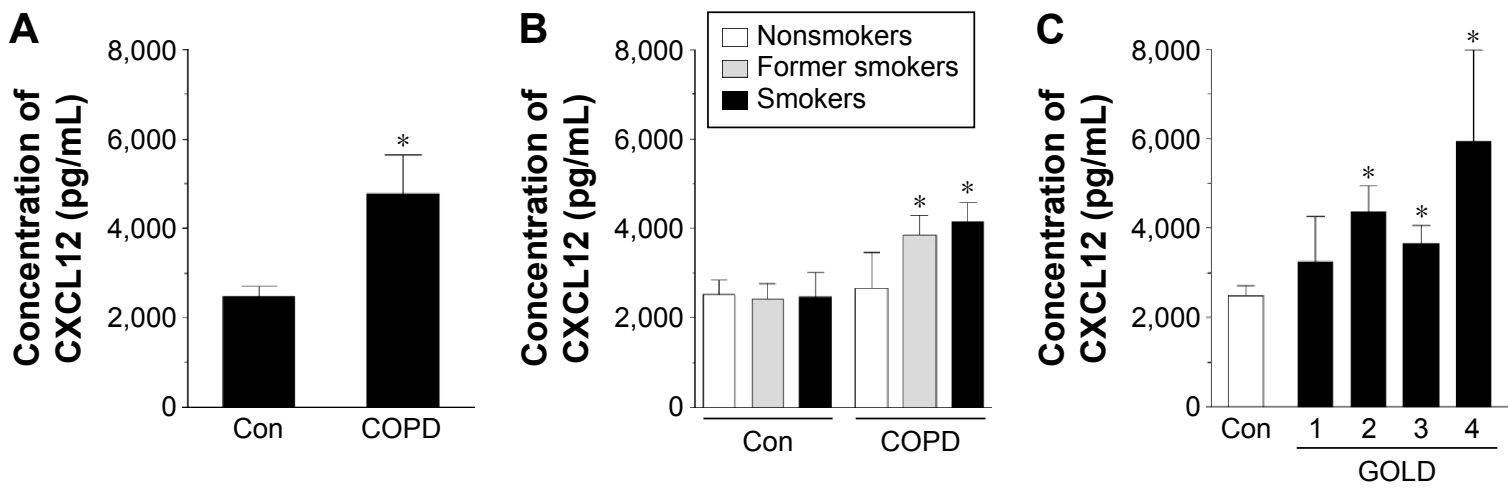

Figure 4 Effect of COPD on the concentration of chemokine CXCLI2 in blood serum.

Notes: The chemokine level was analyzed in serum samples from all patients with COPD and healthy donors (A) as well as from donors divided according to smoking status (B) and obstruction stage (C). The asterisks indicate a significant difference as compared with the Con group. The results are derived from the chemokine measurements in sera obtained from 92 patients with COPD and from 44 healthy volunteers. The results are expressed as mean \pm SEM.

Abbreviations: Con, control; GOLD, Global Initiative for Chronic Obstructive Lung Disease; SEM, standard error of the mean. 
this disease decline significantly in a function of the time that has passed since the patient ceased smoking. ${ }^{23,24}$

As per the GOLD criteria, the level of CCL21 and CXCL12 started to increase in patients in stage 2, 3, and 4 of the disease, that is, patients in whom the obturation became significant. Conversely, the results for patients with early disease (stage 1) were comparable with the healthy controls, suggesting that the severity of COPD corresponds with the promigratory capabilities of the studied chemokines (Figures 2C, 3C, and 4C). This conclusion is in agreement with the observations of other authors who evidenced that lung tumorigenesis is most profound in patients with advanced obturation. ${ }^{25}$

In order to confirm that CCL21 and/or CXCL12 truly contributed to increased migration of the cancer cells in response to serum from patients with COPD, we conducted intervention studies in which CCL21- and CXCL12-dependent signaling was blocked using specific neutralizing antibodies and a specific peptide, respectively. These tests revealed that the efficiency of cancer cell migration was reduced to a level comparable to that of the control group upon neutralization of CCL21 ( $P=0.0002)$. Conversely, when the intervention concerned CXCL12 signaling, the migration of cancer cells remained unchanged (Figure 5).

To the best of our knowledge, our report is the first to show that chemokine CCL21 may contribute to increased migration of A549 cancer cells in actively smoking patients with COPD.

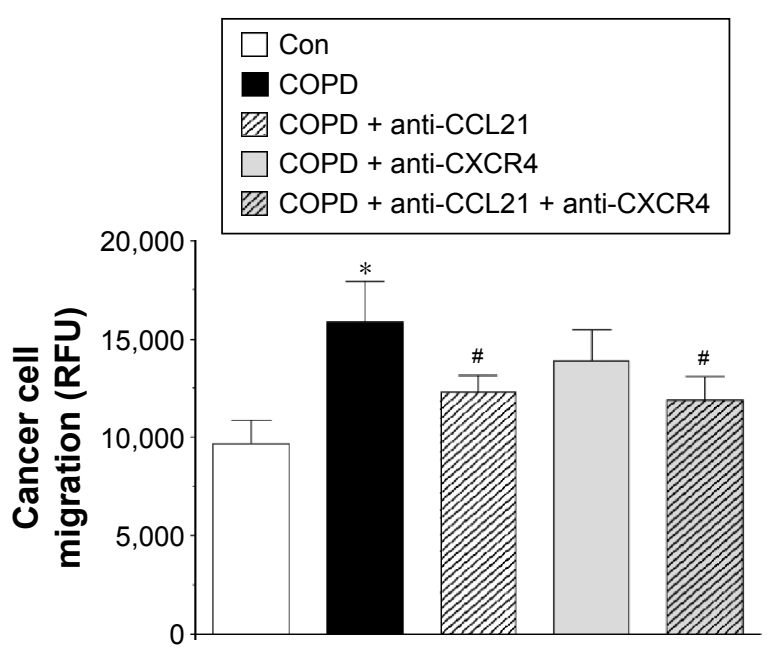

Figure 5 Efficiency of lung cancer cell migration upon neutralization of CCL2I- and CXCLI2-related signaling.

Notes: The asterisk indicates a significant difference as compared with the Con group. The hashes (\#) indicate a significant difference as compared with cells exposed to serum from patients with COPD not subjected to CCL2I/CXCLI2 signaling neutralization. The experiment was performed with sera from 25 patients with COPD and from 25 healthy volunteers. The results are expressed as mean \pm SEM.

Abbreviations: Con, control; RFU, relative fluorescence units; SEM, standard error of the mean.
Taking into account that COPD is a chronic inflammatory process associated with increased secretion and activity of numerous chemokines, for example, CXCL8, CCL2, CXCL5, and CXCL10, ${ }^{7}$ our study provides information on the other chemokines, that is, CCL21 and CXCL12, whose level increases in the course of this pathology. This finding is in line with earlier reports that showed the causative involvement of various COPD-related chemokines in vital aspects of lung cancer cell progression, such as proliferation, ${ }^{26}$ adhesion, ${ }^{21}$ and angiogenesis. ${ }^{27}$ On the other hand, the lack of a full reduction of cancer cell migration to the level in the control group may suggest that there are some other factors (chemokines?) involved in the promigratory capabilities of serum from patients with COPD.

\section{Conclusion}

Taken together, our study identified chemokine CCL21 as an important mediator of increased migration of lung cancer cells in patients with COPD. This finding increases our knowledge on the background of lung cancer development during COPD and, at the same time, points to a new molecular target whose neutralization could be considered an element of management in dealing with this kind of tumor.

\section{Disclosure}

The authors report no conflicts of interest in this work.

\section{References}

1. Young RP, Hopkins RJ, Gamble GD. Clinical applications of genebased risk prediction for lung cancer and the central role of chronic obstructive pulmonary disease. Front Genet. 2012;3:210.

2. Chia CY, Kumari U, Casey PJ. Breast cancer cell invasion mediated by Galpha12 signaling involves expression of interleukins- 6 and -8 , and matrix metalloproteinase-2. J Mol Signal. 2014;9:6.

3. Li Z, Wang Y, Dong S, et al. Association of CXCR1 and 2 expressions with gastric cancer metastasis in ex vivo and tumor cell invasion in vitro. Cytokine. 2014;69(1):6-13.

4. Li Z, Zhang LJ, Zhang HR, et al. Tumor-derived transforming growth factor-beta is critical for tumor progression and evasion from immune surveillance. Asian Pac J Cancer Prev. 2014;15(13):5181-5186.

5. Mak M, Spill F, Kamm RD, Zaman MH. Single-cell migration in complex microenvironments: mechanics and signaling dynamics. J Biomech Eng. 2016;138(2).

6. Hager A, Alexander S, Friedl P. Cancer invasion and resistance. EJC Suppl. 2013;11(2):291-293.

7. Barnes PJ. The cytokine network in chronic obstructive pulmonary disease. Am J Respir Cell Mol Biol. 2009;41(6):631-638.

8. Wang ZL. Association between chronic obstructive pulmonary disease and lung cancer: the missing link. Chin Med J (Engl). 2013;126(1):154-165.

9. Hsu YL, Hou MF, Kuo PL, Huang YF, Tsai EM. Breast tumor-associated osteoblast-derived CXCL5 increases cancer progression by ERK/MSK1/ Elk-1/snail signaling pathway. Oncogene. 2013;32(37):4436-4447.

10. Liu Y, Ji R, Li J, et al. Correlation effect of EGFR and CXCR4 and CCR7 chemokine receptors in predicting breast cancer metastasis and prognosis. J Exp Clin Cancer Res. 2010;29:16. 
11. Berndt B, Haverkampf S, Reith G, et al. Fusion of CCL2 1 non-migratory active breast epithelial and breast cancer cells give rise to CCL21 migratory active tumor hybrid cell lines. PLoS One. 2013;8(5): e63711.

12. Wald O, Izhar U, Amir G, et al. Interaction between neoplastic cells and cancer-associated fibroblasts through the CXCL12/CXCR4 axis: role in non-small cell lung cancer tumor proliferation. J Thorac Cardiovasc Surg. 2011;141(6):1503-1512.

13. Xu Y, Liu L, Qiu X, et al. CCL21/CCR7 promotes G2/M phase progression via the ERK pathway in human non-small cell lung cancer cells. PLoS One. 2011;6(6):e21119.

14. Ben-Baruch A. Organ selectivity in metastasis: regulation by chemokines and their receptors. Clin Exp Metastasis. 2008;25(4):345-356.

15. Han N, Yuan X, Wu H, et al. DACH1 inhibits lung adenocarcinoma invasion and tumor growth by repressing CXCL5 signaling. Oncotarget. 2015;6(8):5877-5888.

16. Miller YE. Pathogenesis of lung cancer: 100 year report. Am J Respir Cell Mol Biol. 2005;33(3):216-223.

17. Friedl $\mathrm{P}$, Wolf K. Plasticity of cell migration: a multiscale tuning model. J Cell Biol. 2010;188(1):11-19.

18. Foster KA, Oster CG, Mayer MM, Avery ML, Audus KL. Characterization of the A549 cell line as a type II pulmonary epithelial cell model for drug metabolism. Exp Cell Res. 1998;243(2):359-366.

19. Katakura Y. Molecular basis for the cellular senescence program and its application to anticancer therapy. Biosci Biotechnol Biochem. 2006; 70(5):1076-1081.

20. Jung MJ, Rho JK, Kim YM, et al. Upregulation of CXCR4 is functionally crucial for maintenance of stemness in drug-resistant non-small cell lung cancer cells. Oncogene. 2013;32(2):209-221.
21. Maekawa S, Iwasaki A, Shirakusa T, et al. Association between the expression of chemokine receptors CCR7 and CXCR3, and lymph node metastatic potential in lung adenocarcinoma. Oncol Rep. 2008;19(6): 1461-1468.

22. Saintigny $\mathrm{P}$, Massarelli E, Lin S, et al. CXCR2 expression in tumor cells is a poor prognostic factor and promotes invasion and metastasis in lung adenocarcinoma. Cancer Res. 2013;73(2):571-582.

23. Fry JS, Lee PN, Forey BA, Coombs KJ. How rapidly does the excess risk of lung cancer decline following quitting smoking? A quantitative review using the negative exponential model. Regul Toxicol Pharmacol. 2013;67(1):13-26.

24. Westmaas JL, Newton CC, Stevens VL, Flanders WD, Gapstur SM, Jacobs EJ. Does a recent cancer diagnosis predict smoking cessation? An analysis from a large prospective US cohort. J Clin Oncol. 2015;33(15): $1647-1652$.

25. Wasswa-Kintu S, Gan WQ, Man SF, Pare PD, Sin DD. Relationship between reduced forced expiratory volume in one second and the risk of lung cancer: a systematic review and meta-analysis. Thorax. 2005; 60(7):570-575.

26. Kawamura M, Toiyama Y, Tanaka K, et al. CXCL5, a promoter of cell proliferation, migration and invasion, is a novel serum prognostic marker in patients with colorectal cancer. Eur J Cancer. 2012;48(14): 2244-2251.

27. Keeley EC, Mehrad B, Strieter RM. Chemokines as mediators of tumor angiogenesis and neovascularization. Exp Cell Res. 2011;317(5): 685-690.
International Journal of COPD

\section{Publish your work in this journal}

The International Journal of COPD is an international, peer-reviewed journal of therapeutics and pharmacology focusing on concise rapid reporting of clinical studies and reviews in COPD. Special focus is given to the pathophysiological processes underlying the disease, intervention programs, patient focused education, and self management protocols.

\section{Dovepress}

This journal is indexed on PubMed Central, MedLine and CAS. The manuscript management system is completely online and includes a very quick and fair peer-review system, which is all easy to use. Visit $\mathrm{http}: / / \mathrm{www}$.dovepress.com/testimonials.php to read real quotes from published authors. 